\title{
Breast reconstruction statistics in Korea from the Big Data Hub of the Health Insurance Review and Assessment Service
}

\author{
Jae-Won Kim, Jun-Ho Lee, Tae-Gon Kim, Yong-Ha Kim, Kyu Jin Chung \\ Department of Plastic and Reconstructive Surgery, Yeungnam University College of Medicine, Daegu, Korea
}

Background Previously, surveys have been used to investigate breast reconstruction statistics. Since 2015, breast reconstruction surgery after mastectomy has been covered by the National Health Insurance Service in Korea, and data from breast reconstruction patients are now available from the Health Insurance Review and Assessment Service (HIRA). We investigated statistics in breast reconstruction in Korea through statistics provided by the HIRA Big Data Hub.

Methods We investigated the number of cases in mastectomy and breast reconstruction methods from April 1, 2015 to December 31, 2016. Data were furnished by the HIRA Big Data Hub and accessed remotely online. Results were tabulated using SAS Enterprise version 6.1.

Results The 31,155 mastectomy cases included 7,088 breast reconstruction cases. Implantbased methods were used in 4,702 cases, and autologous methods in 2,386. The implantbased reconstructions included 1,896 direct-to-implant and 2,806 tissue-expander (2-stage) breast reconstructions. The 2-stage tissue-expander reconstructions included 1,624 expander insertions (first stage) and 1,182 expander-to-permanent-implant exchanges (second stage). Of the autologous breast reconstructions, 705 involved latissimus dorsi muscle flaps, 498 involved pedicled transverse rectus abdominis myocutaneous (TRAM) flaps, and 1,183 involved free-tissue transfer TRAM flaps, including deep inferior epigastric perforator free-tissue transfer flaps. There were 1,707 nipple-areolar complex reconstructions, including 1,565 nipple reconstructions and 142 areola reconstructions. The 1-year mean number of breast reconstructions was 4,050.

Conclusions This was the first attempt to evaluate the total number of breast reconstruction procedures using accurate, comprehensive data, and our findings may prove valuable as a foundation for future statistical studies of breast reconstruction procedures in Korea.

Keywords Mammaplasty / National statistics / National health programs
Correspondence: Jun-Ho Lee Department of Plastic and Reconstructive Surgery, Yeungnam University College of Medicine, 170 Hyeonchung-ro, Nam-gu, Daegu 42415, Korea

Tel: +82-53-620-3482

Fax: +82-53-626-0705

E-mail: junojunho@gmail.com
This work was supported by a 2017 Yeungnam University Research Grant.

This article was presented at the PRS Korea 2017 on November 10-12, 2017 in Seoul, Korea.

\section{INTRODUCTION}

Statistics provide the government with information that can be used for establishing, implementing, and evaluating policies. Using statistics is very important for making decisions in response to social and economic changes [1]. Many countries

Copyright $\odot 2018$ The Korean Society of Plastic and Reconstructive Surgeons

This is an Open Access article distributed under the terms of the Creative Commons Attribution Non-Commercial License (http://creativecommons.org/

licenses/by-nc/4.0/) which permits unrestricted non-commercial use, distribution, and reproduction in any medium, provided the original work is properly cited.

www.e-aps.org 
have national databases to increase the accuracy of statistical data, and collect, process, and publish data to which citizens are provided easy access [2]. Furthermore, countries around the world systematically collect and analyze statistical data from various medical fields and publish them annually. The field of plastic surgery is broad and covers many types of procedures. In recent years, the procedures preferred by patients have changed; statistical data are especially important in identifying such shifts [3-12]. The American Society for Aesthetic Plastic Surgery (ASPS) annually collects procedural statistics on plastic surgery and regularly releases reports showing annual changes in procedural statistics [7]. For its 2017 report, the ASPS collected data by distributing its annual questionnaire to 24,600 plastic surgeons, and the results from a total of 703 surgeons were used for statistical analysis [7]. However, since their findings were based on subjective responses to a questionnaire that was analyzed retrospectively, it may be possible to determine trends more precisely using a complete enumeration.

Korea has a single-payer, universal, and compulsory healthcare insurance system. Most citizens are enrolled in the Korean National Health Insurance (NHI) program, and medical providers are required to submit claims for reimbursement for medical procedures covered by the NHI. Since April 1, 2015, breast reconstruction surgery has been covered by the National Health Insurance Service (NHIS) in Korea, enabling the Health Insurance Review and Assessment Service (HIRA) to collect and store data on the number of breast reconstruction cases. In addition, the HIRA provides a Big Data Hub, which allows researchers to analyze claims data, making it possible to conduct more accurate breast reconstruction studies than previous studies that relied on survey-based statistics [12-17]. The purpose of this study was to analyze statistics in breast reconstruction procedures using objective and reliable statistics obtained through the
HIRA Big Data Hub and to provide basic information on the methods of breast reconstruction used in Korea.

\section{METHODS}

Data on patients diagnosed with breast cancer from April 1, 2015 to December 31, 2016 were obtained from the Big Data Hub of the HIRA. This study was approved by the Institutional Review Board of Yeungnam University Hospital and adhered to the Declaration of Helsinki.

To obtain data from the HIRA, we made an e-mail request for a consultation to use the data for the study. The suitability of the topic, possibility of relevant data extraction, and procedures required to obtain the necessary data were discussed. Then, the researchers were registered, and the topic of the study was submitted to the HIRA, after which the necessary data were extracted. The extracted data can be accessed by visiting one of 40 research centers located in Korea or remotely in a private space via the internet; only one of these two methods may be selected. The service fee for using the Big Data Hub is 50,000 Korean won per day, and institutions receiving research support are eligible to pay $50 \%$ of this fee. Visiting a research center in person posed limitations in terms of accessibility and time constraints; thus, the data were accessed remotely via the internet, as analysis was possible 24 hours per day for the 30 -day study period. It was only possible to access and extract the data for the study after review and approval by the HIRA. The raw data were not made available as they were not fully anonymized; however, the database is programmed to prevent extraction of and access to raw data. To access and extract the necessary information, the data must first be processed; then, the results are submitted for review to ensure the anonymity of the data. When the research was completed, an official document was submitted to notify

\section{Table 1. Main variables in the patient samples}

\begin{tabular}{|c|c|}
\hline Table & Variable \\
\hline Table 20 (general specification) & $\begin{array}{l}\text { Billing statement identification code (key ID), patient ID, provider's ID, stratification variables, strata, age, sex, sample weight, DRG billing } \\
\text { number, claim types, date of admission, insurance type, hospital arrival pathway, major diagnosis, secondary diagnosis, injury from } \\
\text { public service, days of care, initial date of care, final date of care, days in hospitalization, payer's amount, patient's out-of-pocket cost, } \\
\text { total amount, surgical status }\end{array}$ \\
\hline Table 30 (healthcare services) & $\begin{array}{l}\text { Billing statement identification code (key ID), service category, classification type, unit price, total price, daily dosages, days of supply, } \\
\text { quantity of supply, service codes, drug codes }\end{array}$ \\
\hline Table 40 (diagnosis information) & Billing statement identification code (key ID), indicator for major diagnosis, department, diagnosis \\
\hline Table 53 (outpatient prescriptions) & $\begin{array}{l}\text { Billing statement identification code (key ID), classification type, unit price, total price, daily dosages, days of supply, quantity of supply, } \\
\text { service codes, drug codes }\end{array}$ \\
\hline Table of providers & $\begin{array}{l}\text { Provider ID, type of providers, presence of special equipment (CT, MRI, PET), location, number of beds, number of staff per } 50 \text { beds, in- } \\
\text { cluding physicians, dentists, acupuncturists, and nurses }\end{array}$ \\
\hline \multicolumn{2}{|c|}{$\begin{array}{l}\text { IDs were modified to protect private information. } \\
\text { DRG, diagnosis-related group; CT, computed tomography; MRI, magnetic resonance imaging; PET, positron emission tomography. } \\
\text { Reprinted from Kim et al. Epidemiol Health 2014;36:e2014008 [13]. }\end{array}$} \\
\hline
\end{tabular}


the HIRA that the study had been completed. If additional analysis is needed, researchers may apply for an access extension or data retention for a future analysis.

The data analysis of the present study was based on 3,969,419 (T20) data files of billing statements of patients who were diagnosed with breast cancer. Approximately 50,724,479 claims data files (T30) had procedure codes corresponding to healthcare services in each billing statement (Table 1) [13].

We extracted the necessary data by searching and categorizing each procedure code for mastectomy and breast reconstruction according to the Korean Standard Classification of Diseases, Seventh Revision (Table 2). We performed data mining using the procedure codes, selected the patient's procedure code, treatment code, and regional code of the medical institution as variables, and merged the T20 and T30 data files (Fig. 1). The number of mastectomy and breast reconstruction cases was analyzed. In addition, the location of the medical institution, date of the procedure, and insurance type were selected as variables and analyzed. Using data from the billing statements, the number of cases of each type of breast reconstruction was analyzed. The location of medical institutions was categorized by metropolitan cities and provinces.

We examined the number of mastectomy cases, the number of breast reconstruction cases, the number of cases of immediate
Table 2. Inclusion criteria for the study based on procedure codes through insurance payment

\begin{tabular}{|ll|}
\hline Code & \multicolumn{1}{c|}{ Procedure } \\
\hline N7130 & $\begin{array}{c}\text { Radical mastectomy (including modified radical mastectomy and } \\
\text { radical breast-conserving operations; without axillary lymph node } \\
\text { dissection) }\end{array}$ \\
N7131 & $\begin{array}{c}\text { Simple mastectomy } \\
\text { N7132 }\end{array}$ \\
Subcutaneous mastectomy \\
N7135 & $\begin{array}{c}\text { Radical mastectomy (including modified radical mastectomy and } \\
\text { radical breast-conserving operations) }\end{array}$ \\
N7140 & Autologous - LD muscle - LD flap \\
N7141 & Autologous - LD muscle - muscle-sparing LD myocutaneous flap \\
& (thoracodorsal artery perforator flap) \\
N7142 & Autologous - LD muscle - extended LD myocutaneous flap \\
N7143 & Autologous - pedicled TRAM flap \\
N7144 & Autologous - bipedicled TRAM flap \\
N7145 & Autologous - transverse TRAM free flap \\
N7146 & Autologous - muscle sparing TRAM free flap \\
N7147 & Autologous - deep inferior epigastric artery perforator free flap \\
N7148 & Implant based - expander insertion (2-stage breast reconstruction, \\
& first stage) \\
N7149 & Implant based - DTI \\
N7150 & Implant based - expander to permanent breast implant (2-stage \\
& breast reconstruction, second stage) \\
N7152 & Nipple-areolar reconstruction - nipple reconstruction \\
N7153 & Nipple-areolar reconstruction - areolar reconstruction \\
\hline LD, latissimus dorsi; TRAM, transverse rectus abdominis musculocutaneous; DTI, \\
direct-to-implant. \\
\hline
\end{tabular}

\section{Fig. 1. Process of data mining}

Data mining algorithm of the provided data based on billing statements from the Health Insurance Review and Assessment Service Big Data Hub.

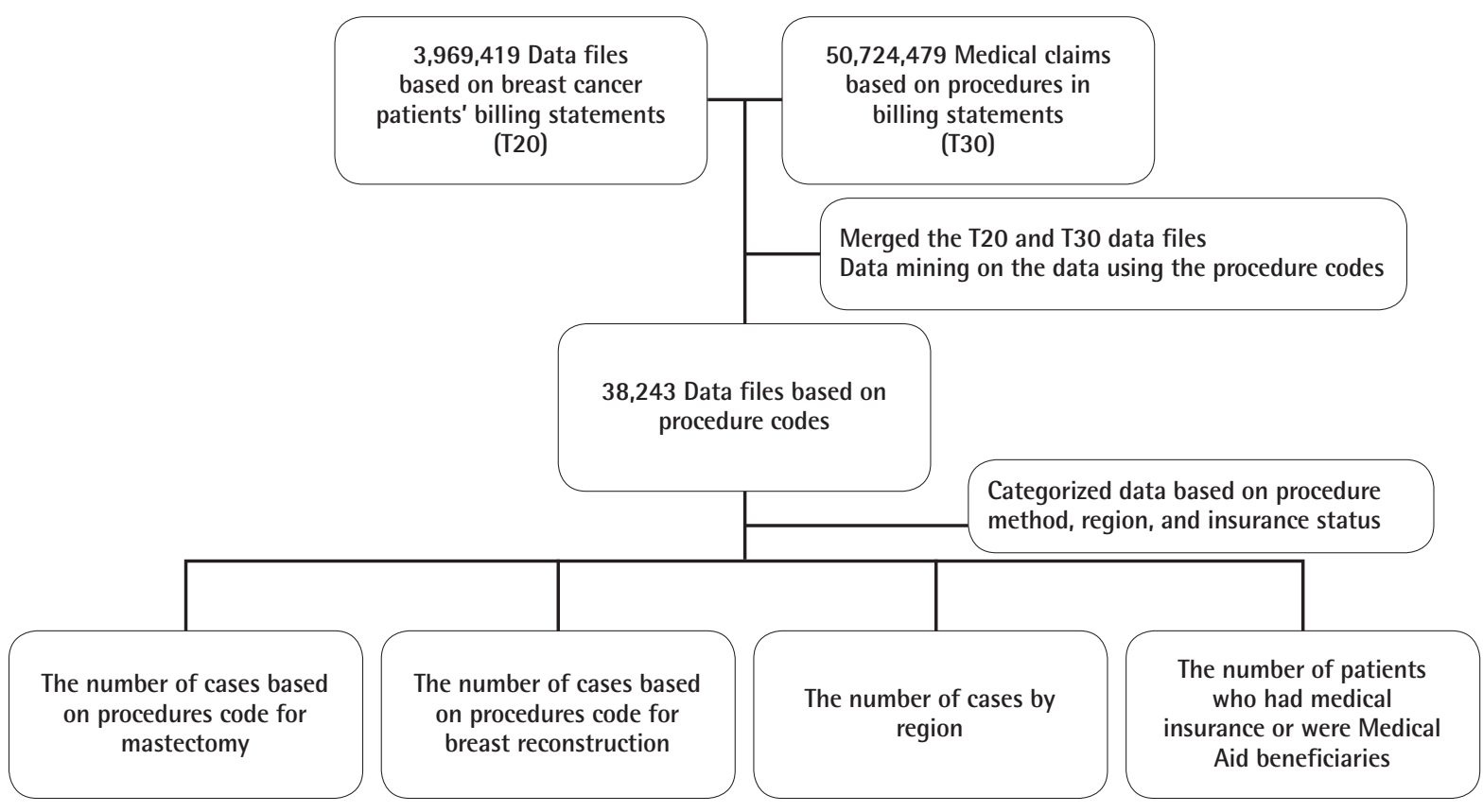


breast reconstruction using implants, the number of cases of breast reconstruction using autologous tissue, and the number of cases of delayed breast reconstruction using expanders. The data were analyzed with SAS Enterprise version 6.1 (SAS Institute Inc., Cary, NC, USA); Excel (Microsoft Corp., Redmond, WA, USA) was used to organize and analyze the data.

\section{RESULTS}

We examined claims data from all patients with breast cancer. There were a total of 31,155 claims with a mastectomy procedure code. Of these, 29,785 involved radical mastectomy, 1,294 involved simple mastectomy, and 76 involved subcutaneous mastectomy (Table 3).

A total of 7,088 claims were submitted for breast reconstruction, including 2,386 cases of autologous breast reconstruction (33.66\%) and 4,702 cases of implant-based breast reconstruction (66.34\%). Of the 2,386 autologous cases, 705 (29.54\%) used a latissimus dorsi (LD) flap, 498 (20.87\%) used a pedicled

\section{Table 3 . The number of mastectomy cases by type of}

\section{surgery}

\begin{tabular}{|lc|}
\hline Methods & No. of cases \\
\hline Total & 31,155 \\
Radical mastectomy (including modified radical mastectomy & 6,786 \\
$\quad \begin{array}{l}\text { and radical breast-conserving operations; without axillary } \\
\text { lymph node dissection) }\end{array}$ \\
$\begin{array}{l}\text { Simple mastectomy } \\
\text { Subcutaneous mastectomy }\end{array}$ \\
$\begin{array}{l}\text { Radical mastectomy (including modified radical mastectomy } \\
\text { and radical breast-conserving operations) }\end{array}$ & 22,999 \\
\hline
\end{tabular}

Table 4. The number of cases of breast reconstruction by method

\begin{tabular}{|lc|}
\hline Methods & No. of cases \\
\hline Autologous & 2,386 \\
LD & 705 \\
pTRAM & 498 \\
fTRAM (fTRAM and DIEP) & 1,183 \\
Implant-based methods & 4,702 \\
Expander insertion (first stage) & 1,624 \\
DTI & 1,896 \\
Expander to permanent breast implant (second stage) & 1,182 \\
Total & 7,088 \\
Nipple-areolar complex & \\
Nipple reconstruction & 1,565 \\
Areolar reconstruction & 142 \\
\hline LD, latissimus dorsi; pTRAM, pedicled transverse rectus abdominis myocutane- \\
ous; fTRAM, free-tissue transfer transverse rectus abdominis myocutaneous; \\
DIEP, deep inferior epigastric perforator; DTI, direct-to-implant. \\
alfTRAM includes the total number of cases for simple fTRAM, muscle-sparing \\
fTRAM, and DIEP. \\
\hline
\end{tabular}

transverse rectus abdominis myocutaneous (pTRAM) flap, and 1,183 (49.58\%) used a free-tissue transfer transverse rectus abdominis myocutaneous (fTRAM) flap. The category of fTRAM flaps encompassed simple fTRAM, muscle-sparing fTRAM, and deep inferior epigastric perforator free-tissue transfer flaps. Of the 4,702 cases using implant-based methods, 1,896 (40.32\%) were direct-to-implant (DTI) and 2,806 (59.68\%) involved expander use. Of the cases of implant-based reconstruction using an expander, 1,624 (34.54\%) involved expander insertion (first stage), and 1,182 (25.14\%) involved the exchange of an expander for a permanent breast implant (second stage). The total number of nipple-areolar complex (NAC) reconstruction cases was 1,707 , of which 1,565 (91.68\%) were nipple reconstructions, while 142 (8.32\%) were areolar reconstructions (Table 4).

Data from all reconstruction types over the entire study period were divided by the number of years to obtain 1-year mean values. The 1-year mean values by breast reconstruction type are as follows. Of the 4,050 annualized cases of breast reconstruction, $1,364(33.66 \%)$ were autologous breast reconstructions, and 2,686 (66.34\%) were reconstructions via implant-based methods. Of the 1,364 cases of autologous breast reconstruction, 403 (29.54\%) involved an LD flap, 285 (20.87\%) involved a pTRAM flap, and 676 (49.58\%) involved an fTRAM flap. Of the 2,686 annualized cases of breast reconstruction using an implant-based method, 1,083 (40.32\%) were DTI, and 1,603 (59.68\%) were reconstructions using an expander, including 928 (34.54\%) expander insertions (first stage) and 675 (25.14\%) exchanges of an expander for a permanent breast implant (second-stage). The 975 NAC reconstructions included 894 nipple reconstructions (91.69\%) and 81 areolar reconstruc-

Table 5 . The 1-year mean number of cases of breast reconstruction by type

\begin{tabular}{|lc|}
\hline Methods & No. of cases \\
\hline Autologous & 1,364 \\
LD & 403 \\
PTRAM & 285 \\
fTRAM (fTRAM and DIEP)a) & 676 \\
Implant-based methods & 2,686 \\
Expander insertion (first stage) & 928 \\
DTI & 1,083 \\
Expander to permanent breast implant (second stage) & 675 \\
Total & 4,050 \\
Nipple-areolar complex & 894 \\
Nipple reconstruction & 81 \\
Areolar reconstruction & \\
\hline LD, latissimus dorsi; pTRAM, pedicled transverse rectus abdominis myocutane- \\
ous; fTRAM, free-tissue transfer transverse rectus abdominis myocutaneous; \\
DIEP, deep inferior epigastric perforator; DTI, direct-to-implant. \\
alfTRAM includes the total number of cases for simple fTRAM, muscle-sparing \\
fTRAM, and DIEP. \\
\hline
\end{tabular}


Table 6. The number of patients covered by medical insurance or Medical Aid (1-year mean)

\begin{tabular}{|lcccc|}
\hline & Breast reconstruction & Mastectomy & Total population of Korea & P-value ${ }^{\text {a) }}$ \\
\hline Medical insurance & $3,963(97.84)$ & $17,155(96.37)$ & $50,425,000(97.1)$ & $<0.05^{\text {b) }}$ \\
Medical Aid & $87(2.16)$ & $647(3.63)$ & $1,506,000(2.9)$ & \\
Total & 4,050 & 17,802 & $51,931,000$ & \\
\hline
\end{tabular}

Values are presented as number (\%).

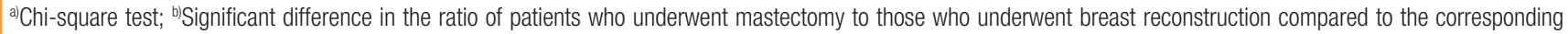
ratio in the total population of Korea.

tions (8.31\%) (Table 5).

Of the 4,050 cases of breast reconstruction, $87(2.16 \%)$ were performed in Medical Aid beneficiaries and 3,963 (97.84\%) in patients with medical insurance. Of the 17,802 cases of mastectomy, 17,155 (96.37\%) had medical insurance and 647 (3.63\%) were Medical Aid beneficiaries (Table 6). The ratio of mastectomy procedures to breast reconstruction procedures among Medical Aid beneficiaries was lower than that among patients with medical insurance $(\mathrm{P}<0.05)$.

The analysis by region showed that Seoul had the highest total number of breast reconstruction cases $(n=4,256)$, as well as the highest number of autologous breast reconstruction cases $(\mathrm{n}=1,456)$ and implant-based breast reconstruction cases $(\mathrm{n}=2,800)$. Incheon and Gyeonggi-do had the second-highest total number of breast reconstruction cases $(n=1,128)$, as well as the second-highest number of autologous breast reconstruction cases $(n=430)$ and implant-based breast reconstruction cases $(n=698)$. Busan, Ulsan, and Gyeongsangnam-do had the third-highest number of breast reconstruction cases $(n=894)$, as well as the third-highest number of autologous $(n=256)$ and implant-based breast reconstruction cases $(n=638)$. Daegu and Gyeongsangbuk-do had the fourth-highest total number of breast reconstruction cases $(n=303)$, the fourth-highest number of autologous breast reconstruction cases $(n=113)$, and the fifth-highest number of implant-based breast reconstruction cases. Daejeon and Chungcheongnam-do had the fifth-highest total number of breast reconstruction cases $(n=258)$, the fifthhighest number of autologous breast reconstruction cases $(n=65)$, and the fourth-highest number of implant-based breast reconstruction $(\mathrm{n}=193)$ (Table 7).

\section{DISCUSSION}

Korea has a unified, universal NHI system that covers approximately $98 \%$ of the total population. The HIRA processes claims data from 46 million patients annually, corresponding to $90 \%$ of the total population. As of 2011, there are approximately 80,000 healthcare providers in Korea. Claims data from the HIRA include diagnosis information, treatment types, procedure types,
Table 7. The number of cases by region

\begin{tabular}{|lrrr|}
\hline Regions $^{\text {a) }}$ & Autologous $^{\text {b) }}$ & Implant $^{\text {c) }}$ & Total \\
\hline Seoul & 1,456 & 2,800 & 4,256 \\
Incheon, Gyeonggi-do & 430 & 698 & 1,128 \\
Busan, Ulsan, Gyeongsangnam-do & 256 & 638 & 894 \\
Daegu, Gyeongsangbuk-do & 113 & 190 & 303 \\
Daejeon, Chungcheongnam-do & 65 & 193 & 258 \\
Gwangju, Jeollanam-do & 28 & 81 & 109 \\
Jeollabuk-do & 16 & 46 & 62 \\
Chungcheongbuk-do & 9 & 45 & 54 \\
Gangwon-do & 11 & 10 & 21 \\
Jeju-do & 2 & 1 & 3 \\
Total & 2,386 & 4,702 & 7,088 \\
\hline
\end{tabular}

LD, latissimus dorsi; pTRAM, pedicled transverse rectus abdominis myocutaneous; fTRAM, free-tissue transfer transverse rectus abdominis myocutaneous; DIEP, deep inferior epigastric perforator; DTI, direct-to-implant.

a) Regions divided by province; 'b)Autologous procedures included LD, pTRAM, fTRAM, and DIEP; ${ }^{\text {cII }}$ mplant-based procedures included DTI, expander insertion (first stage) and expander to permanent implant (second stage).

surgical records, and prescriptions; these data provide valuable information for medical service research. HIRA data are collected when a healthcare provider and medical consumer request reimbursement for healthcare services provided in accordance with NHIS policies. The annual number of Korean patients who submit health insurance claims is approximately 46 million; the HIRA provides national-level data collected from the healthcare institutions throughout Korea that match the number of claims submitted by patients [13].

Most previous statistical data on plastic surgery procedures were from retrospective survey analyses or included a small sample; thus, the reliability of these reports varies. However, breast reconstruction procedures are now covered by the NHI, making it possible to obtain an accurate count of these procedures using the data made available by the HIRA.

From April 1, 2015 to December 31, 2016, the total number of breast reconstruction cases was 7,088, corresponding to $22.75 \%$ of the 31,155 mastectomy cases. According to the ASPS statistical report, the number of breast reconstructions in the USA in 2000,2015 , and 2016 was $78,832,106,338$, and 109,256 , respectively. These numbers increased by $39 \%$ from 2000 to 2016, and by $3 \%$ from 2015 to 2016 [7]. In addition, 
the number of breast reconstruction procedures per 100 mastectomies increased by approximately $67 \%$ from 2009 (24 breast reconstructions per 100 mastectomies) to 2014 (40 breast reconstructions per 100 mastectomies) [9]. This indicates that breast reconstruction procedures are still not performed as often in Korea as in the USA.

The 1-year mean number of breast reconstruction cases in Korea was 4,050 based on the available HIRA data. Of these, 1,364 (33.66\%) cases involved autologous methods, while 2,686 (66.34\%) involved implant-based methods. The ratio of autologous to implant-based methods was roughly $2: 1$. According to the ASPS statistics, a total of 109,256 breast reconstruction cases were performed in the USA in 2016. Of these procedures, 20,650 (18.9\%) involved autologous tissue reconstruction and 88,606 (81.1\%) involved implant-based reconstruction [7]. The ratio of autologous to implant-based methods was much higher in the USA (1:4) than in Korea (1:2). In the USA, the percentage of patients who received breast reconstruction after mastectomy was found to be roughly $40 \%$, indicating that breast reconstruction procedures are still performed more often in the USA than in Korea. Of the total number of breast reconstruction procedures in the USA $(109,256)$, the proportion of bilateral reconstructions was higher $(n=71,399$ [65.35\%] $)$ than that of unilateral reconstructions $(n=30,324$ [34.65\%]) [7]. This may be explained by the fact that prophylactic mastectomy procedures are performed relatively frequently in the USA, but are rarely performed in Korea. In such cases, the implant-based method is preferred over autologous tissue reconstruction because the implant-based method is less invasive, makes it easier to match the shapes of the left and right breasts, has minimal downtime, and does not have donor site problems, such as donor site morbidity.

In total, 88,606 cases of implant-based breast reconstruction were performed in the USA in 2016 [7]. Of these, 79,019 (89.18\%) involved a tissue expander, and 9,587 (10.82\%) were implant-only procedures, yielding a ratio of approximately 9:1. Of the 2,686 implant-based breast reconstructions performed in Korea, 1,083 (40.32\%) were DTI, and 1,603 (59.68\%) involved a tissue expander, corresponding to a ratio of approximately 2:3. Thus, the ratio of DTI to tissue-expander procedures was higher in Korea than the ratio of implant-only procedures to tissue-expander procedures in the USA. This may be attributed to the fact that single-stage expander insertion is common in Korea, and can also be explained by patients' desire for a quick return to their social routines. Differences in surgical approach, such as the amount of skin spared in flap harvesting, may also affect this ratio.

According to statistics provided by the Breast Cancer Society of Korea, the number of breast reconstruction procedures performed in 2000 was 99, which increased by 11 times to 1,111 in $2013[11,18]$. In addition, 1,279 breast reconstruction procedures were performed in 2014, indicating that a significant increase took place recently [11]. When comparing the total number of cases of autologous and implant-based reconstruction with the 1-year mean (4,050 cases), it can be seen that the number of breast reconstruction procedures has risen significantly over a year. This increase in the number of procedures performed may have partially been due to changes in public awareness about breast reconstruction and the recent coverage of this procedure by the NHI. Another explanation may be that the statistical findings of previous studies were not accurate. Additionally, for patients who require implant replacement due to an infection or capsular contracture, NHI provides coverage using the procedure code for breast reconstruction. The procedure code used in such cases is that for an expander-to-permanent-implant exchange, and each patient may be covered 2 or more times by insurance for the procedure, which may result in an overestimation of the number of patients receiving breast reconstruction.

The number of breast reconstruction procedures by insurance type was also analyzed. In 2016, 2.9\% (1,506,000) of the total population of Korea $(51,931,000)$ were Medical Aid beneficiaries and $97.1 \%(50,425,000)$ had medical insurance through the NHIS [19]. The 1-year mean number of mastectomies was 17,802; 647 (3.63\%) of these were performed in Medical Aid beneficiaries and 17,155 (96.37\%) were performed in patients with medical insurance. Of the 4,050 breast reconstruction procedures, 87 (2.16\%) were performed in Medical Aid beneficiaries and 3,963 (97.84\%) were performed in patients with medical insurance. The ratio of the number of medical insurance patients who underwent breast reconstructions to the number of medical insurance patients among the total population was significantly higher than the ratio of the number of Medical Aid beneficiaries who underwent breast reconstructions to the number of Medical Aid beneficiaries among the total population. Additionally, the ratio of the number of patients who underwent breast reconstruction to the number of patients who underwent mastectomy was significantly higher among patients with medical insurance than among Medical Aid beneficiaries. This difference between insurance types may be due to financial burdens in receiving breast reconstruction, even when the medical cost is covered by the NHI.

The regional distribution of breast reconstruction cases was analyzed. The region with the highest number of breast reconstructions performed was Seoul, with 4,256 cases (60.05\%), accounting for more than $50 \%$ of the total procedures performed 
$(\mathrm{n}=7,088)$. Of the procedures performed in Seoul, 1,456 (20.54\%) were autologous breast reconstructions, and 2,800 (39.5\%) were implant-based reconstructions. The ratio of the autologous method to the implant-based method was 1:2. Thus, implant-based reconstructions were performed more often than autologous reconstructions. This ratio was similar to the overall ratio of 1:2. The region with the second-highest number of breast reconstructions performed was Incheon and Gyeonggido with 1,128 (15.91\%). Of the procedures performed in Incheon and Gyeonggi-do, 430 (6.06\%) were autologous reconstructions, and 698 (9.85\%) were implant-based reconstructions. The ratio of autologous reconstructions to implant-based reconstructions was about $1: 1.6$, and the ratio of autologous methods performed in this region was higher than the overall ratio. The region with the third-highest number of breast reconstruction procedures performed was the Busan, Ulsan, and Gyeongsangnam-do region with 894 (12.61\%) procedures. Of these procedures, $256(3.61 \%)$ were autologous reconstructions, and 638 (9\%) were implant-based reconstructions. The ratio of autologous reconstructions to implant-based reconstructions was roughly $1: 2.6$, which was higher than the overall ratio. The region with the fourth-highest number of procedures performed was the Daegu and Gyeongsangbuk-do region, with 303 (4.27\%). Of these procedures, 113 (1.59\%) were autologous reconstructions, and 190 (2.68\%) were implant-based reconstructions. The number of procedures dramatically decreased from the third-highest region to the fourth-highest region. Additionally, the ratio of autologous reconstructions to implant-based reconstructions in the Daegu and Gyeongsangbuk-do region was $1: 1.68$, which was higher than the overall ratio.

Seoul, Incheon, and Gyeonggi-do are geographically close and part of the greater Seoul metropolitan area. Thus, medical care services tend to be concentrated in these regions. Of the 7,088 total cases of breast reconstruction, 5,384 (75.96\%) were performed in these three regions. Additionally, considering that the total population of the Seoul, Incheon, and Gyeonggi-do regions is $23,979,019$, corresponding to $46.77 \%$ of the total population of Korea, the distribution of breast reconstruction procedures was concentrated in the greater Seoul metropolitan area. Based on reimbursement requests by location of the medical institution, patients living in other regions appear to commute to metropolitan areas to receive treatment.

The present study has some limitations. We were unable to obtain information on the number of breast reconstruction procedures that were performed following lumpectomy or partial breast mastectomy. In addition, the procedure codes in the data provided were not comprehensive enough for other types of tissue flap procedures to be counted. Thus, it was difficult to dis- tinguish between immediate and delayed procedures. Moreover, the period in which the data were collected was short. This may have resulted in counting errors, since some of the patients who underwent a 2-stage procedure using tissue expanders were not counted. This would have included those patients who underwent the first stage of the procedure before it was covered by the $\mathrm{NHI}$, and those who underwent the first stage, but not the second stage, of the procedure within the data collection timeframe. Due to the nature of the data provided, patients' electronic medical records could not be accessed, making it difficult to conduct a more sophisticated analysis. Finally, information on breast reconstructions performed prior to April 1, 2015 was not available from the HIRA Big Data Hub database. In addition, because of the limited time period from which data are available from the HIRA Big Data Hub database, only patients diagnosed in 2 consecutive years (from April 1, 2015 to December 31,2016$)$ were included and yearly trends in breast reconstruction could not be analyzed. Therefore, breast reconstruction trends should be further evaluated in future studies. Nonetheless, the results of this study are meaningful in that this was the first attempt to assess the number of breast reconstruction procedures in Korea using accurate and comprehensive data from the Big Data Hub.

The number of plastic breast reconstruction cases is increasing globally, and our findings obtained by analyzing the breast reconstruction statistics provided by the HIRA indicate that the number of cases is continuously growing in Korea as well, with most procedures performed in the Seoul and Gyeonggi-do regions. Our study utilized the HIRA Big Data Hub to obtain the most objective and accurate procedural statistics. These data may be valuable as a foundation for future studies of breast reconstruction trends in Korea. Finally, this study was the first attempt to evaluate the total number of breast reconstruction procedures using accurate, comprehensive data.

\section{NOTES}

\section{Conflict of interest}

No potential conflict of interest relevant to this article was reported.

\section{Ethical approval}

The study was approved by the Institutional Review Board of Yeungnam University Hospital (IRB No. YUMC 2017-03-010) and performed in accordance with the principles of the Declaration of Helsinki. Written informed consents were obtained. 


\section{ORCID}

Jun-Ho Lee https://orcid.org/0000-0002-0062-6420

Tae-Gon Kim https://orcid.org/0000-0002-6738-4630

Yong-Ha Kim https://orcid.org/0000-0002-1804-9086

Kyu Jin Chung https://orcid.org/0000-0001-6335-1818

\section{REFERENCES}

1. Nilsen K. The impact of information policy: measuring the effects of the commercialization of Canadian government statistics. Westport: Ablex Publishing; 2001.

2. Clarkson KW, Meiners RE. Government statistics as a guide to economic policy: food stamps and the spurious increase in the unemployment rates. Policy Rev 1977;(1):27.

3. Rugiu PS, Sykes PJ. A history of plastic surgery. Berlin: Springer; 2007.

4. Heidekrueger PI, Juran S, Ehrl D, et al. Global aesthetic surgery statistics: a closer look. J Plast Surg Hand Surg 2017;51: 270-4.

5. Gurunluoglu R, Gurunluoglu A, Williams SA, et al. Current trends in breast reconstruction: survey of American Society of Plastic Surgeons 2010. Ann Plast Surg 2013;70:103-10.

6. Rohrich RJ. The increasing popularity of cosmetic surgery procedures: a look at statistics in plastic surgery. Plast Reconstr Surg 2000;106:1363-5.

7. Plastic surgery statistics report [Internet]. Arlington Heights, IL: American Society of Plastic Surgeons, c2017 [cited 2018 Feb 13]. Available from https://www.plasticsurgery.org/ documents/News/Statistics/2016/plastic-surgery-statisticsfull-report-2016.pdf.

8. Breast cancer facts \& figures [Internet]. Atlanta, GA: American Cancer Society; c2015 [cited 2018 Feb 16]. Available from: https://www.cancer.org/content/dam/cancer-org/research/ cancer-facts-and-statistics/breast-cancer-facts-and-figures/ breast-cancer-facts-and-figures-2015-2016.pdf.

9. Breast reconstruction surgery for mastectomy in hospital inpatient and ambulatory settings, 2009-2014 [Internet]. Rockville, MD: Agency for Healthcare Research and Quality; c2017 [cited 2018 Feb 16]. Available from: https://www. ncbi.nlm.nih.gov/books/NBK481368/pdf/Bookshelf_
NBK481368.pdf.

10. Jagsi R, Jiang J, Momoh AO, et al. Trends and variation in use of breast reconstruction in patients with breast cancer undergoing mastectomy in the United States. J Clin Oncol 2014;32:919-26.

11. Breast cancer facts \& figures [Internet]. Seoul: Korean Breast Cancer Society; c2016 [cited 2018 Feb 18]. Available from: http://www.kbcs.or.kr/journal/file/2016_Breast_ Cancer_Facts_and_Figures_updated.pdf.

12. Park YT, Yoon JS, Speedie SM, et al. Health insurance claim review using information technologies. Healthc Inform Res 2012;18:215-24.

13. Kim L, Kim JA, Kim S. A guide for the utilization of Health Insurance Review and Assessment Service National Patient Samples. Epidemiol Health 2014;36:e2014008.

14. Joo SY, Goo YK, Ryu JS, et al. Epidemiology of trichomoniasis in South Korea and increasing trend in incidence, Health Insurance Review and Assessment 2009-2014. PLoS One 2016;11:e0167938.

15. Yhim HY, Jang MJ, Bang SM, et al. Incidence of venous thromboembolism following major surgery in Korea: from the Health Insurance Review and Assessment Service database. J Thromb Haemost 2014;12:1035-43.

16. Kang S, Kim HS, Choi ES, et al. Incidence and treatment pattern of extremity soft tissue sarcoma in Korea, 20092011: a nationwide study based on the Health Insurance Review and Assessment Service Database. Cancer Res Treat 2015;47:575-82.

17. Son H, Park J, Song SH, et al. Rapid increase of health care utilization and cost due to benign prostatic hyperplasia in Korean men: retrospective population-based analysis using the Health Insurance Review and Assessment service data. J Korean Med Sci 2015;30:180-5.

18. Yi M, Joung WJ, Park EY, et al. Decision making experience on breast reconstruction for women with breast cancer. J Korean Acad Nurs 2016;46:894-904.

19. 2016 Medical benefit statistics [Internet]. Wonju: National Health Insurance Service; c2017 [cited 2018 Feb 16]. Available from: http://www.nhis.or.kr/menu/boardRetriveMenuSet. xx?menuId=F3323. 